\title{
The Importance of Daily Fast Fresh Cadaver Dissection (How can we organize it?)
}

\begin{abstract}
Objectives: Recently a world famous neurosurgeon admitted at the world congress: the mortality rate of the most difficult tumor was $18 \%$ when he started to operate and the next 5 year went down under $5 \%$. We don't like to speak about it. It is the dark side of science. How can we avoid these complications? "Learning curve" is a "politically correct" expression, but at what cost? A lot of suffering, death, complications behind it. Do we need to improve our skills on living patients? Is it ethical? How many complicated operation/year do we need to maintain our skills on high level? Do we have enough training laboratory in hospitals? It is not available in thousands of mid centers.
\end{abstract}

Methods: In the last 15 years we have gradually introduced the daily fresh cadaver dissections in our practice in 3 mid centers. We examine the difficulties which had to be faced in centers which have no anatomy lab.

Results: The ethical and practical difficulties were negotiable, but the organization were not easy.

Discussion: We should change of the postgraduate training methodology in intra and extracranial micro and endoscipic and endovascular surgery return to daily training like in sport. Fresh cadavers are available in every hospital. This would be the main basis of the „nil nocere” surgical prinicpal.

Keywords: fresh cadaver, daily fast fresh cadaver practice, neurosurgical training

Volume 9 Issue I - 2019

\author{
Csókay András, Attila Josvai, Csókay Gergely, \\ Jäckel Márta \\ Hungarian Defence Forces Medical Center, Hungary
}

\begin{abstract}
Correspondence: Csókay András M.D.Ph.D. Hungarian Defence Forces Medical Center, Budapest ,44.Robert K. str, I 34 Hungary, Email csokaya@gmail.com
\end{abstract}

Received: July 15, 2018 | Published: January 08, 2019

\section{Introduction}

Medical diagnostic developments in the last fifty years are undoubtably a great benefit for the patients. Unfortunately this is also a great loss for surgeons wanting to practice or gain experience from fresh cadaver work, which has practically disappeared in developed countries. The autopsy has become unnecessary. Cause of death and pathological processes can be determined most of the time without it. There are many microsurgical disciplines on the head (neurosurgery, ENT, maxillofacial, eye/orbit) that can not be learned from assistance alone. Such surgeries are practically done by a single surgeon (e.g. general microsurgical -hand surgery, and endoscopic -abdominal or orthopaedic operations). Complications during deep brain or internal and external cranial base surgeries are very hard to correct, if they can be corrected at all. Large vessel injuries, or complications during an abdominal, or even an open cardiac surgery pose also difficulties, but there is some time to collect thoughts, outline a surgical strategy, or call for a more experienced surgeon, therefore these complications are correctable by new suturing or repair without further ischemic damage. However, during deep brain or external and internal skull base approaches (esp. if those are trans-nasal, trans-orbital, trans maxillo-facial), micro- and endoscopic and endovascular operations, micro vessel injuries or neuronal structure injuries are impossible to correct.

The current structure for gaining experience and preventing complications in the surgical field is to learn from more experienced masters and perform more and more difficult operative steps. Doctors who have time and opportunity to take part in hands on courses have also more chance to practice, especially if it is in the same hospital where they are employed.

What can those do, who don't have these opportunities in hundred thausend of mid centers? It is also important as hundreds of articles ${ }^{1}$ pointed out, that anatomical workshops with chemically conserved or prior frozen cadavers serve a good alternative, but not lifelike to work on. In addition, there are some cadaver models trying to emulate circulation, but access to such models is very limited. ${ }^{1-5}$ What happens in real life? Young neurosurgeons gain experience most of the time by lots of assisting in operations, then having their first own surgical experiences on patients during on call, often during night time hours, and finally have their own elective surgeries. Many doctors also send difficult patients to other clinics with more experience. Consequently, all these factors decrease the quality of care for the rest of patients. This is also a psychological problem for doctors who cause the complication, and as a result, they do not accept elective patients, creativity and motivation decreases, which leads to further frustration. The goal is to develop a method to prevent this outcome. It is well known that musicians and athletes don't practice during performances and competitions, nor only one-two times per month. We tried to develop a daily training regimen in cooperation with the Pathology Ward. Daily fresh cadaver practice can model even challenging operations, and may prevent the aforementioned complications. In our concept, the basis for complication avoidance is not yearly workshops, monthly or weekly but cadaver practice on daily basis, and within this frame the simulation of vascular and nerve injuries in difficult-to-reach locations (such as internal and external skull base, or ventricles of the brain), where vascular bypass grafts and nerve 
grafts can be made. Another advantage of our concept is improving surgical orientation beyond the borders of the classical microsurgical exposures, which further facilitates the interdisciplinary teamwork between the specialties through targeted, fast exposures. Furthermore, modelled tumour removal can also be practiced by placing a piece of autologue tissue (mimikling the tumour itself) from the opposite side, allowing the practicing doctors to remove it from a regular exposure. These can be applied to a broad spectrum in the field of microsurgical specialities such as neuro-, ENT-, maxillofacial surgery, or ophthalmology.

Regarding the daily fresh cadaveric practice, the further important questions have to be raised/answered :

i. How can 75-90 minute sessions be integrated into the daily routine of the Patholgy Ward with the least interference?

ii. Is there a legal, ethical barrier to cadaver practices? Can grieving relatives be asked for permission?

iii. Are fresh (6-24 hour) cadavers more lifelike than preserved anatomical or frozen cadavers?

\section{Method}

We tried to answer these questions with these observation

i. We studied the labour process in autopsy room.

ii. In many countries, permission from family members of patient with brain death, as a potential organ donor for transplantation, is not required. Can autopsy be required when the cause of death is diagnosed and radiologically known? Probably not.

iii. Differences between fresh and frozen / chemically preserved cadavers with regards to tissue plasticity and venous bleeding was noted.

\section{Results}

i. It seemed to be optimal to perform cadaver practices in the early morning or the late afternoon when the work of the Pathology Ward is not disturbed.

ii. Getting permission from family members is legally difficult, time consuming and most of the time is refused. In Hungary according to a 1997 law autopsy can be performed without permission for four reasons: criminal, clinical, scientific or educational. Looking at the dead body as a whole, the extent of tissue damage during an autopsy is overwhelming in comparison to microsurgical practice: the trunk is opened in the midline, from upper thorax all the way down to the pelvis, all the organs are removed, and dissected to $1 \mathrm{~cm}$ slices. In addition, the whole convexity of the skull (fronto-temporo-parietally) is circumferentially craniotomized, and the brain is removed, and finally cut to slices. During closure, the cranial cavity is filled with paper, the convexity of the skull is readjusted, all the organs are replaced into the abdominal cavity, and the skin is closed. The regular dissection of the human body including the brain and skull base are a significantly coarser intervention than the microsurgical operations. (For example careful retraction of the brain or endonasal endoscopic manipulations). Due to the these reasons, the hospital management and the Ethics Committee have no objection to cadaver practice surgery carried out within an autopsy. iii. Tissue plasticity was preserved in fresh (not frozen) cadavers for 1-2 days. Venous bleeding was also conserved, which seems to be one of the main benefits compared to chemically conserved anatomical or frozen cadaveric models. Physical characteristics of the tissues in fresh cadavers are lifelike, which is plays a significant role when performing micro-vascular anastomosis.

Daily fresh cadaver practice greatly refines anatomical orientation (esp. during endoscopic practice), improves fine motor skills when considering microsurgical dissection, or microanastomosis.

\section{Conclusion}

All over the world, the famous masters and schools advise cadaver workshops and often require them before starting work in specific areas (e.g., endoscopic surgery or bypass). The reality is that no one questions the legitimacy of anatomical workshops, due to a high degree of usefulness. The new proposal will add a special practical consideration, which may lead to further professional development. We have made over 3000 (first author), (the second more than 600) practice cadaver operations in the past 14 years to model the most difficult surgical situations. Just as in sports, or in art of music performance the monthly or weekly practice . is not sufficient. Daily practice in fresh cadaver exercises is crucial. Neurosurgeons can perform 100-150 difficult operations yearly can be thankful, but it is hard to reach for even big center leaders. Most have only 30-50 very difficult operations. Most of these are performed during duty hours. This is explained by the fact that patients gravitate toward doctors with more experience. Daily cadaver practice can elevate this number above 300 so lots of experience can be gained in spite of relatively low number of patients.

Lots of benefits could be gained from the following:

1. Primarily severe acute patients who arrive at night during duty hours, stand a higher chance of recieving exeptional treatment.

2. Professionals performing microsurgeries on the head (like neuro, eye, ENT, maxillofacial) also benefit, because the psychological toll of not being able to control difficult situations due to inexperience decreases.

3. Also the field of microsurgery benefits because more doctors can take part in practice sessions leading to the development of more talent and the development of the whole field.

Based on years of experience our hypothesis is, that professionals practicing this psychologically and physically difficult profession will see a great decrease in complications with cadaver work. Even in contraindicated cases (complex cranio- cervical, external and internal cranial base pathologies, large tumours in $80+$ year old patients where the duration of op. very important) good results can be achieved. In one particular case 29 inductive fresh cadaver practice operations were performed before the final (successful) operation. Many presentations were held and one article was written in the past three years about free of charge Hungarian and international daily 60-80 minute cadaver practice. The goal is to reach as many countries and professionals performing microsurgeries as possible.

Daily cadaver practice can be psychologically burden some. However spiritual activities (prayer, meditation) can alleviate one's mental tension (Figure 1). 


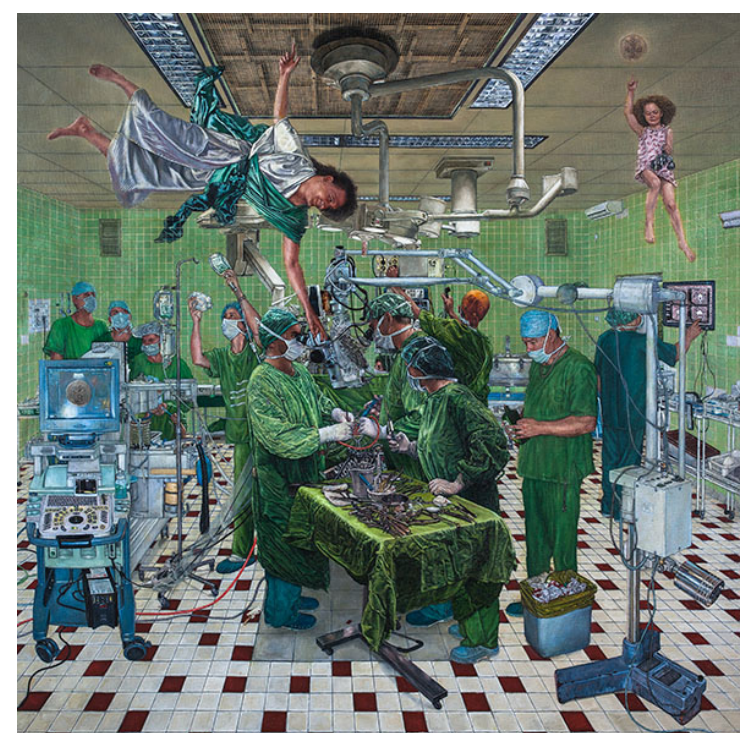

Figure I Daily fresh cadaver exersises.

\section{Acknowledgments}

Special thanks to the artist, Muhari-Papp Sándor Balázs for medical illustration.

\section{Conflicts of interest}

The author declares no conflicts of interest.

\section{References}

1. Aboud E, Al-Mefty O, Yaşargil MG. New laboratory model for neurosurgical training that simulates live surgery. $J$ Neurosurg. 2002;97(6):1367-1372.

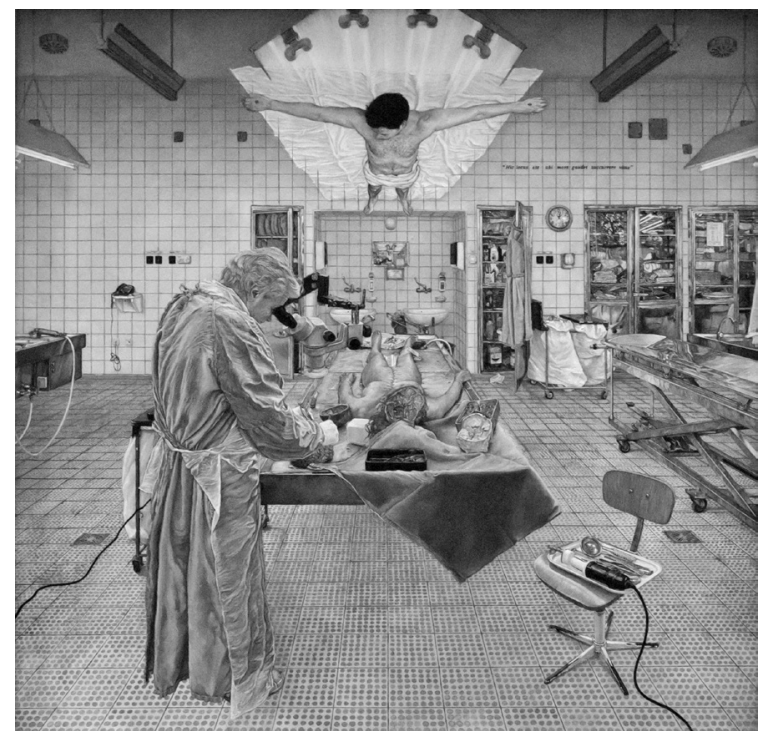

2. Hamamcioglu MK, Hicdonmez T, Tiryaki M, et al. A laboratory training model in fresh cadaveric sheep brain for microneurosurgical dissection of cranial nerves in posterior fossa. British J Neurosurg. 2008;22(6):769777 .

3. John PC. Modern approaches to teaching and learning anatomy. BMJ. 2008;337:310.

4. Sanan A, Aziz K, Janjua R, et al. Colored silicone injection for use in neurosurgical dissections: Anatomic technical note. Neurosurgery. 1999;45(5):1267-1268

5. Hicdonmez T, Hamamcioglu MK, Parsak T, et al. A laboratory training model for interhemispheric-transcallosal approach to the lateral ventricle. Neurosurg Rev. 2006;29(2):159-162. 